\title{
A Dynamic Ferroelectric Capacitance Model for Circuit Simulators
}

\author{
C. Kühn ${ }^{1}$, H.Hönigschmid ${ }^{2}$, O. Kowarik ${ }^{1}$, E. Gondro ${ }^{1}$ and K. Hoffmann ${ }^{1}$ \\ ${ }^{1}$ Institute of Electronics, ET4, Universtität der Bundeswehr München \\ Werner-Heisenberg-Weg 39, 85577 Neubiberg, Germany \\ ${ }^{2}$ Infineon AG, Balanstr. 73, 81541 München, Germany
}

\begin{abstract}
A dynamic model has been developed that is based on the Preisach approach for ferroelectric capacitors. It accounts for different switching reaction of the dipoles dependent on the applied voltage gradients. A method for extracting the necessary parameters by measuring the coercive voltage versus applied frequency is provided. The model was verified by testing capacitors of different geometries. Excellent agreement was achieved between our measurements and the corresponding circuit simulations. The simulation of a FeRAM-cell with our new dynamic model shows that the bit line signal decreases substantially with fast read/write times.
\end{abstract}

\section{INTRODUCTION}

The Preisach-Everett model, described e.g. in [1], has proven to be of great value for the use in circuit simulators [2].

So far, however, very few attention has been paid to include time dependent behavior into the Preisach model.

The ferroelectric hysteresis curve shows a strong frequency dependence: The coercive voltage $V_{C}$ increases with the frequency $f$ of the external electric field. This was already stated by Merz [3] and measured by Mihara [4] for $\mathrm{SrBi}_{2} \mathrm{Ta}_{2} \mathrm{O}_{9}$ and $\mathrm{Pb}(\mathrm{Zr}, \mathrm{Ti}) \mathrm{O}_{3}$. Liu [5] derived this frequency dependence of the hysteresis curve from the energy dissipation during one period of domain reversal. Lohse [6,7] also measured a frequency dependent $V_{C}$ and attributed this phenomenon to the reversible movement of domain walls.

A first model for circuit simulators that included switching time dependence was presented by Takeo [9] using the response function derived by Fatuzzo and Merz [8]. The spontaneous polarization, however, is modeled by a simple decay function. Therefore this model can not account for minor hysteresis loops. Another approach for dynamic modeling of ferroelectric capacitors was made by Rep and Prins [10], who replaced the ferroelectric capacitor by an equivalent circuit of non-linear resistors and capacitors.

\section{MEASUREMENT OF HySTERESIS CURVES}

Measurement was performed by using strontiumbismuth-tantalate (SBT) capacitors of different sizes
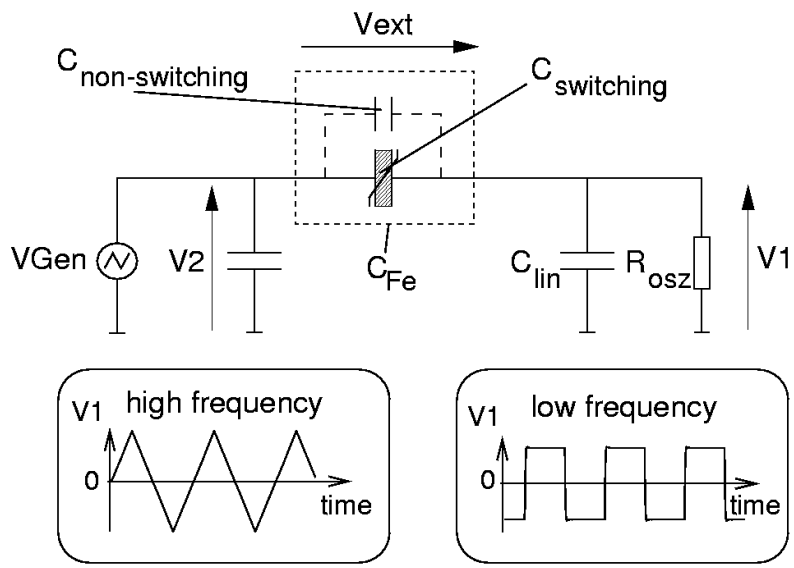

Fig. 1: Sawyer-Tower circuit: For high frequencies $(1 \mathrm{kHz}-3 \mathrm{MHz}) V_{l}$ is dominated by the charge on $C_{l i n}$, whereas in the low frequency range ( $1 \mathrm{~Hz}-3 \mathrm{~Hz}) V_{l}$ is governed by the current through $R_{\text {osz }}$.

$\left(100 \mu \mathrm{m}^{2}, 400 \mu \mathrm{m}^{2}\right.$ and $\left.1000 \mu \mathrm{m}^{2}\right)$. The polarization on the ferroelectric capacitor was established with a SawyerTower-Circuit (Fig.1). Both the voltage $V_{l}$, representing the polarization charge of the FE-capacitor, and the voltage $V_{2}$, corresponding to the output voltage $V_{G e n}$ of the generator, are measured with identical cable lengths at the same digital oscilloscope. Because of this symmetry differences in the signal running time are eliminated

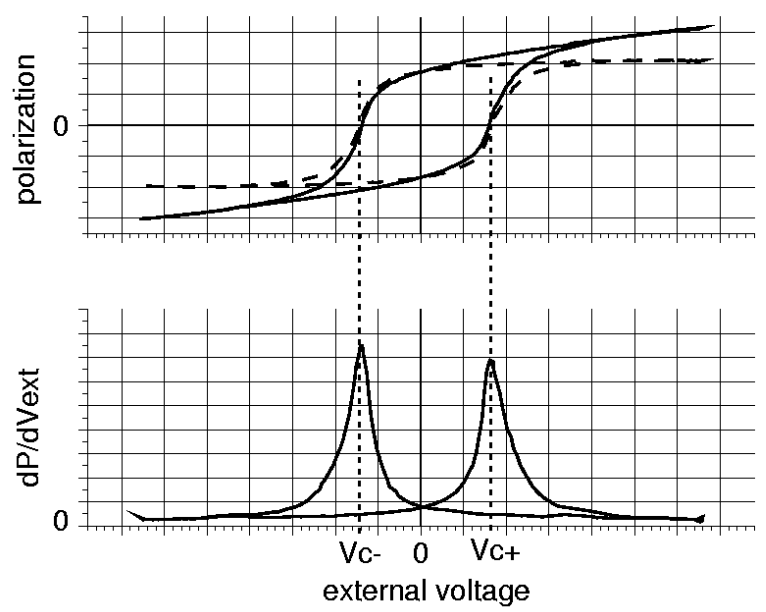

Fig. 2: The upper curve shows a typical measurement of the hysteresis curve for $\mathrm{f}=20 \mathrm{kHz}$ (solid line). The maximum of its derivative $\mathrm{dP} / \mathrm{dV}_{\text {ext }}$ (lower curve) coincides with the coercive voltage of the hysteresis curve without linear contribution (dashed line / upper curve). 


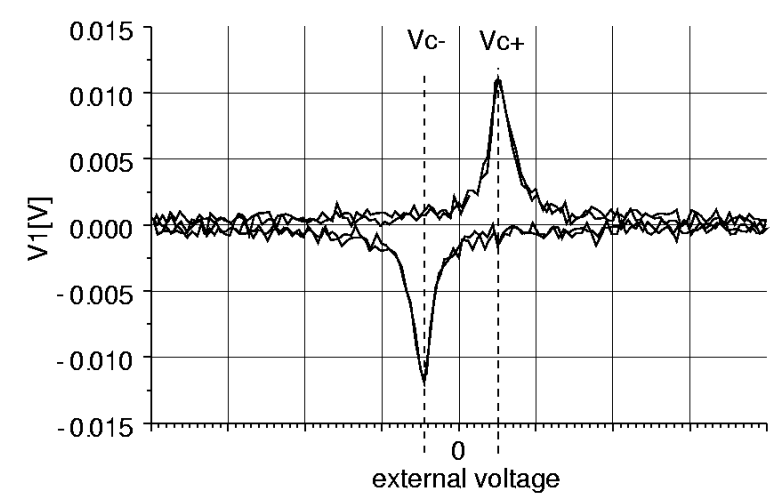

Fig. 3: Typical measurement of $V_{C}$ at low frequencies $(f=2 \mathrm{~Hz})$. The maximum of $\mathrm{V}_{1}$ indicates the current maximum which is at the place of the coercive voltage (for $\mathrm{f}<5 \mathrm{~Hz}$ ).

providing thus a reliable determination of the coercive voltage $V_{C}$ up to $3 \mathrm{MHz}$. For high frequencies $(1 \mathrm{kHz}-$ $3 \mathrm{MHz}) V_{l}$ is dominated by the charge on $C_{\text {lin }}$, whereas in the low frequency range $(1 \mathrm{~Hz}-3 \mathrm{~Hz}) V_{l}$ is governed by the current through $R_{o s z}$.

The applied pulses had triangular shape with an amplitude of $4 \mathrm{~V}$, guaranteeing that the FE material is driven into full saturation. The applied frequency was varied from $1 \mathrm{~Hz}$ to $3 \mathrm{MHz}$.

The coercive voltage $V_{C}$ is established by determining the maximum of $d Q_{F E} / d V_{e x t}$ of the hysteresis loop $Q_{F E}\left(V_{e x t}\right)$, where $V_{e x t}$ is the external voltage across $C_{F E}$ (Fig.2). Thus the linear contribution to the polarization charge that stems from the non-switching part of the FEcapacitance does not influence the determination of $V_{C}$.

In the high frequency range the maximum of $d Q_{F E} / d V_{e x t}$ can be determined directly from the $Q_{F E}\left(V_{e x t}\right)$-curve. The position of this maximum is not affected by $R_{o s z}$ at frequencies $\mathrm{f} \geq 1 \mathrm{kHz}$.

For low frequencies the following equation holds:

$$
\frac{d Q_{F E}}{d V_{e x t}}=\frac{I}{d V_{2} / d t-d V_{1} / d t}=\frac{(1 / R) \cdot V_{1}}{\text { const }-d V_{1} / d t}
$$

Due to the triangular shape of $V_{G e n}$ the derivative of $V_{2}$ is a

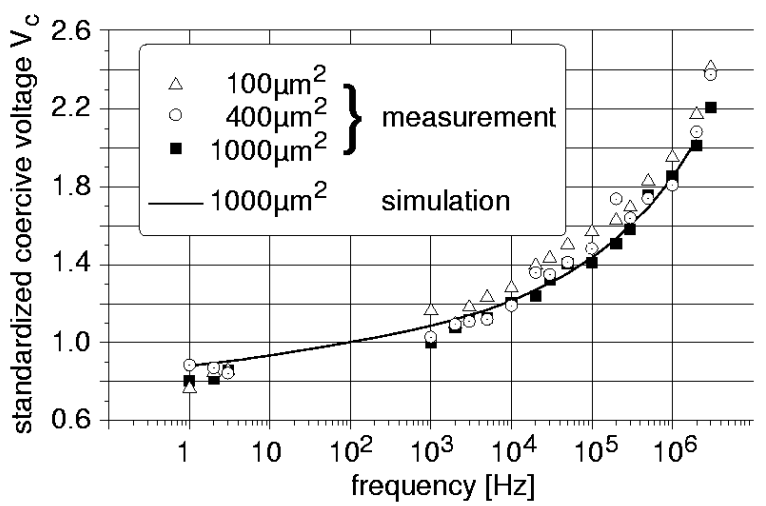

Fig. 4: Measurements $\left(100 \mu \mathrm{m}^{2}\right.$ : triangles, $400 \mathrm{~m}^{2}$ : circles, $1000 \mu \mathrm{m}^{2}$ : squares) and simulation $\left(1000 \mu \mathrm{m}^{2}\right.$ : line) of the coercive voltage dependent on the applied frequency. Standard: $V_{C}$ at $1 \mathrm{kHz}$ for $1000 \mu \mathrm{m}^{2}$. constant. For frequencies below $5 \mathrm{~Hz} \quad V_{C}$ can be determined directly by $V_{l}$, as $d V_{l} / d t \leq 5 \%$. const. Fig. 3 shows a typical measurement.

Fig. 4 shows the results of our measurements: A slight shift to higher $V_{C}$ values can be observed for smaller capacitor areas.

THE MODEL

\section{The original Preisach model}

The basic idea of the Preisach model is, that the ferroelectric material consists of a set of individual dipoles that contribute to the total polarization. Each of these dipoles has two individual coercive voltages, $v_{c+}$ and $v_{c-}$, that are required to make it switch in the opposite direction. Thus each dipole has a rectangular hysteresis loop. Based on the assumption that the dipoles are noninteracting the hysteresis loop of the macroscopic system is seen as a superposition of these hysteresis units.

$v_{c+}$ and $v_{c-}$ of the hysteresis units in the macroscopic system are statistically distributed.

The distribution of the dipoles to their up and down states is dependent on the applied voltage and the course it has taken. This means that the turning points of the voltage curve are of great importance for the dipole distribution [2].

The total polarization $P$ of the ferroelectric results in:

$$
P\left(V, V_{1}, V_{2}, \ldots\right)=\iint_{v_{C+} v_{C-}} \rho\left(v_{C+}, v_{C-}\right) D\left(V, V_{1}, V_{2}, \ldots\right) \cdot p d v_{C+} d v_{C-}
$$

$p$ is the polarization of a single dipole, $\rho\left(v_{c+}, v_{c-}\right)$ the density function of the dipoles with respect to their individual coercive voltages and $D$ a direction operator $( \pm 1)$ depending on the actual voltage and the turning points it has passed.

The tanh-function is a useful approximation for the integral in (2) when $\rho$ is taken as a Gaussian distribution:

$$
P\left(V_{e x t}\right)=A \cdot P_{\text {Sat }} \cdot \tanh \left[a\left(V_{e x t} \mp V_{C \pm}\right)\right]
$$

$P_{S a t}$ is the saturation polarization and $V_{C \pm}$ is the macroscopic coercive voltage, i.e. the mean value of the individual coercive voltages. The behavior of the non saturation loops are taken into account by a factor, so that $A \cdot P_{\text {Sat }}$ represents that proportion of dipoles that take part in the switching process of the non-saturation loop. This is the well-known model described by Bo Jiang [2].

\section{Dynamic model}

In the model above the switching of the dipoles followed immediately to the applied voltage pulse. The polarization reversal, however, is not a immediate process, but has a certain delay time. Fatuzzo and Merz investigated the mechanisms of the reversal of spontaneous polarization [8]. They used pulse measurements for determining the 


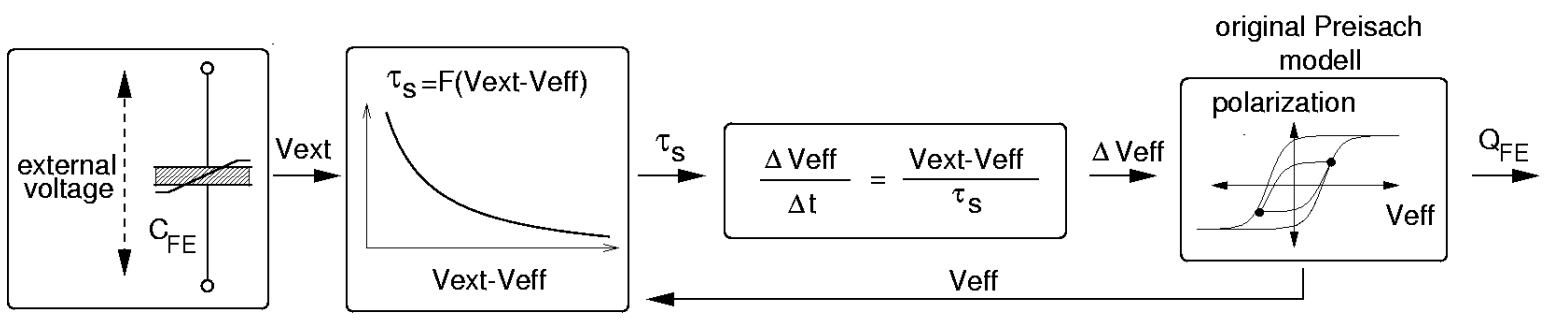

Fig. 5: Schematic view of the implementation of a switching time function $\tau_{\mathrm{S}}=\mathrm{F}\left(\mathrm{V}_{\mathrm{ext}}-\mathrm{V}_{\text {eff }}\right)$ into the Preisach model for circuit simulators.

switching time of the ferroelectric polarization. Switching time $\tau_{\mathrm{S}}$ is defined here as the time during which a current flows due to polarization reversal. The following relationship between switching time $\tau_{S}$ and applied field $E_{\text {ext }}$ was found for weak fields:

$$
\tau_{S}=\tau_{\infty} \exp \left(\alpha / E_{e x t}\right)
$$

It is attributed to the nucleation of domains of the opposite orientation and justified by thermodynamic reasoning $[8,11]$. For higher fields the growth of already existing domains is the preponderate mechanism:

$$
\tau_{S}=\frac{d}{\mu \cdot E_{\text {ext }}}
$$

Here $d$ is the thickness of the crystal sample and $\mu$ is the domain wall mobility.

For the sake of generality we assume the following relationship between switching time and applied voltage:

$$
\tau_{S}=F\left(V_{e x t}\right)
$$

where $F\left(V_{e x t}\right)$ is a function that will be discussed closer in the next section. At this point we will not specify the mechanisms that lead to the delay in switching time. Be it caused by the nucleation of domains and consequent propagation of their walls in the crystal [8] or by the reversible movement of domain walls hindered by internal fields as stated in [7].

For the implementation of this time delay an effective voltage $V_{e f f}(t)$ is calculated from the applied voltage $V_{\text {ext }}(t)$. A simple relaxation formulation is used:

$$
\frac{d V_{e f f}}{d t}=\frac{V_{e x t}(t)-V_{e f f}(t)}{\tau_{S}}
$$

The Preisach model itself is left unchanged but the calculated $V_{\text {eff }}(t)$ is applied to it (cf. Fig.6). So (3) now becomes:

$$
P\left(V_{\text {eff }}\right)=A \cdot P_{\text {Sat }} \cdot \tanh \left[a\left(V_{\text {eff }} \mp V_{C \pm}\right)\right]
$$

The dipoles whose individual coercive voltages $v_{C \pm}$ are within the voltage step $\Delta V_{\text {eff }}$ will switch in the corresponding time step $\Delta t$. The macroscopic coercive voltage $V_{C \pm}$ in (8) can now be seen as the "static coercive voltage", which of course is not correct as the switching itself is a dynamic process. So it is reasonable to see $V_{C \pm}$ just as another parameter.

The voltage argument in (6) is modified to

$$
\tau_{s}=F\left(V_{e x t}-V_{e f f}\right)
$$

We assume that the switching time $\tau_{S}$ is only dependent on the difference between the applied voltage $V_{\text {ext }}$ and the coercive voltage $v_{C}$ of the individual dipoles. The switching is modeled by $V_{e f f}(t)$ and takes place when $V_{e f f}=v_{C}$. So $V_{e f f}(t)$ indicates the individual coercive voltages of the dipoles in the Preisach model and the difference $V_{\text {ext }}-V_{\text {eff }}$ represents the potential barrier that has to be passed when the individual dipoles switch to the other state.

As $\tau_{S}=F\left(V_{\text {ext }}-V_{\text {eff }}\right)$ is seen as a function only of voltages a constant voltage gradient would cause the same switching time delay for all dipoles:

$$
d V_{\text {ext }} / d t=\text { const } \Rightarrow d V_{\text {eff }} / d t=d V_{\text {ext }} / d t=\text { const }
$$

\section{EXTRACTION OF PARAMETERS}

Unfortunately it is not possible to generate a constant voltage gradient with our measurement circuit (Fig. 1) as the voltage drop on the linear capacitor influences the voltage $V_{\text {ext }}$ across the FE-capacitor. So another method to determine the function $\tau_{S}=F\left(V_{e x t}-V_{e f f}\right)$ and its parameters has to be used:

We measure the coercive voltage dependent on the frequency $V_{C}(f)$, which is defined as the external voltage, at which the polarization charge $\mathrm{Q}_{\mathrm{Fe}}$ equals zero:

$$
V_{e x t}=V_{C}(f) \Leftrightarrow Q_{F e}\left(V_{e f f}\right)=0
$$

With (8) and $V_{2}=V_{e x t}+V_{l}$ and $V_{l}=Q_{F E} / C_{\text {lin }}$ (cf. Fig.1), the following conditions are valid, with the assumption that no discharging of the linear capacitor takes place:

$$
\begin{aligned}
& Q\left(V_{\text {eff }}\right)=0 \Rightarrow V_{\text {eff }}=V_{C \pm} \\
& V_{1}=0 \\
& V_{2}=V_{\text {ext }}=V_{C}(f)
\end{aligned}
$$

$V_{C \pm}$ is the static coercive voltage. At the coercive voltage (7) can be expressed with (9) and $\Delta V:=V_{C}(f)-V_{C}$ :

$$
\left.\frac{d V_{e f f}}{d t}\right|_{V_{e f f}=V_{C}}=\frac{V_{e x t}-V_{e f f}}{F\left(V_{e x t}-V_{e f f}\right)}=\frac{\Delta V}{F(\Delta V)}
$$

The time derivative of the voltage across the linear capacitor is (cf. 8):

$$
\frac{d V_{1}}{d t}=\frac{Q_{0}}{C_{\text {lin }}} \cdot \frac{a}{\cosh ^{2}\left\{a\left(V_{\text {eff }}-V_{C}\right)\right\}} \cdot \frac{d V_{e f f}}{d t}
$$

At the coercive voltage (14) becomes with (13):

$$
\left.\frac{d V_{1}}{d t}\right|_{V_{\text {eff }}=V_{C}}=\frac{Q_{0} a}{C_{\text {lin }}} \cdot \frac{\Delta V}{F(\Delta V)}
$$

For the determination of $F(\Delta V)$ it has to be kept in mind that at lower frequencies the gradient of $V_{l}$ is affected by the discharging of the linear capacitor $\mathrm{C}_{\text {lin }}$. We took this 


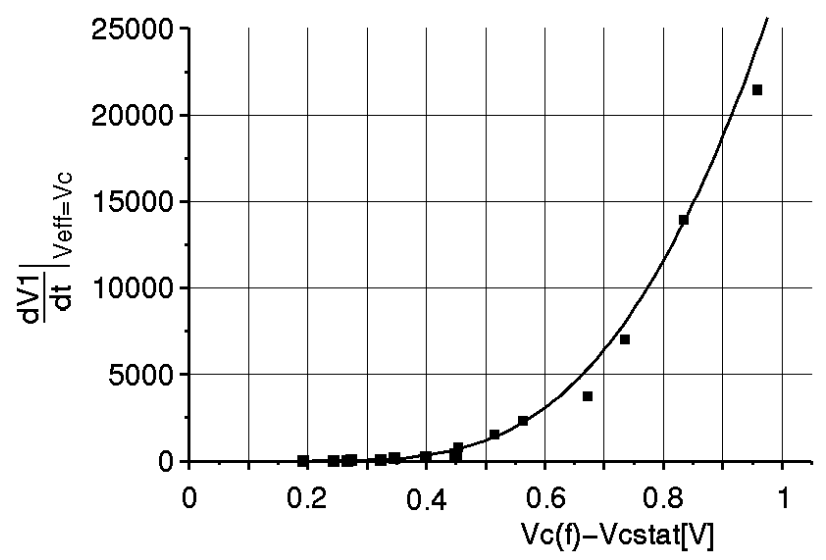

Fig. 6: The function $\tau_{\mathrm{S}}=\mathrm{F}\left(\mathrm{V}_{\mathrm{ext}}-\mathrm{V}_{\mathrm{eff}}\right)$ is determined by the measured voltage gradient $\mathrm{dV}_{1} / \mathrm{dt}$ vs. $\Delta \mathrm{V}=\mathrm{V}_{\text {ext }}-\mathrm{V}_{\text {eff }}$ (15). (squares: measured data, line: fitted curve).

discharging into consideration by a correction factor for the measured voltage gradient. This correction factor was established by simulation of the discharging process. Fig. 6 shows the results of our measurements.

For the fitted curve (4) was used, which suggests that nucleation of domains is the preponderate mechanism in our circuit. But it has to be pointed out, that other functions $F(\Delta V)$ can be used should they prove to be of greater accuracy.

\section{IMPLEMENTATION TO SIMULATOR}

The Preisach model without switching time delay is implemented in a circuit simulator by storing the voltage turning points [2]. By these turning points the amounts of dipoles in the up- and down-states are unambiguously defined, so that also the course of non-saturation loops can be modeled accurately.

The dynamic model is implemented according to (4) and (7). The derivatives are replaced by difference quotients. Fig. 4 shows the results for the simulated frequency dependent coercive voltage compared with our measurements of the Sawyer-Tower circuit.

\section{APPLICATION: FERAM}

With our new dynamic model predictions for FeRAMcells in memory arrays can be performed. Fig. 7 shows simulations where a $1 \mu \mathrm{m}^{2}$-FeRAM cell is evaluated at a bit line with a $100 \mathrm{fF}$ parasitic capacitor. For writing different write times were used. The plate is held at a constant $1.5 \mathrm{~V}$, while the states " 0 " or " 1 " are written to the FeRAM-cell by pulsing the Bit line. The cell is evaluated by precharging the Bit line to $0 \mathrm{~V}$ and consequent discharging of the FeRAM-cell. The read and write times are varied between $10 \mathrm{~ns}$ and $5 \mu \mathrm{s}$ caused a decline of the signal distance for the fast read/write cycles. This has to be taken into consideration for accurate modeling of low voltage FeRAM-arrays with fast $\mathrm{read} / \mathrm{write}$ times.

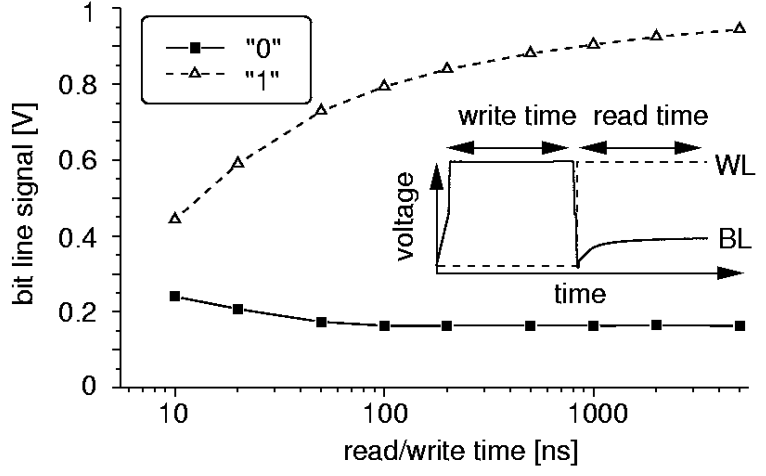

Fig. 7: Simulation of a FeRAM-cell. The difference of the Bit line signals for the state " 0 " and " 1 " increases with increasing duration of the read/write time.

\section{CONCLUSION}

The observed increase of coercive voltage in hysteresis curves can be modeled by a dynamic model. It is an improvement of the standard Preisach model that was refined by introducing a delay time. An extraction method for the necessary parameters was presented. The characterized delay time function corresponds to switching time functions established earlier in literature. This new model was implemented in the circuit simulator and showed good agreement with measurement. The dynamic modeling of the switching time for the polarization reversal is imperative for accurate prediction of FeRAM-performance.

\section{REFERENCES}

[1] I. Mayergoyz, Mathematical Models of Hysteresis, Springer, New York, 1991

[2] B. Jiang et al., "Computationally Efficient Ferroelectric Capacitor Model for Circuit Simulation", Symposium on VLSI Technology, 1997, pp. 141-142

[3] W. Merz, "Domain Formation and Domain Wall Motion in Ferroelectric $\mathrm{BaTiO}_{3}$ Single Crystals", Physical Review, Vol. 95/3, 1954, pp. 690-698

[4] T. Mihara et al., "Characteristics of Bismuth Layered $\mathrm{SrBi}_{2} \mathrm{Ta}_{2} \mathrm{O}_{9}$ Thin-Film Capacitors and Comparison with $\mathrm{Pb}(\mathrm{Zr}, \mathrm{Ti}) \mathrm{O}_{3}$ ”, Jpn. J. Appl. Phys., Vol. 34, 1995, pp. 5233-5239

[5] J.-M. Liu et al., "Frequency Response and Scaling of Hysteresis for Ferroelectric $\mathrm{Pb}\left(\mathrm{Zr}_{0.52}, \mathrm{Ti}_{0.48}\right) \mathrm{O}_{3}$ Thin Films Deposited by Laser Ablation", Journal of Appl. Phys., Vol. 86/9, 1999, pp. 5198-5202

[6] O. Lohse et al., "Temperature Dependence of the Polarization Reversal in Lead-Zirconate-Titanate Thin Film Capacitors", ISAF 1998, pp. $27-30$

[7] O. Lohse et al., "Reversible and Irreversible Contributions to the Polarization in $\mathrm{SrBi}_{2} \mathrm{Ta}_{2} \mathrm{O}_{9}$ Ferroelectric Capacitors", Ferroelectric Thin Films VI Symposium. Mater. Res. Soc. 1998, pp. 267-278

[8] E. Fatuzzo and W. Merz, "Switching Mechanism in Triglycine Sulfate and Other Ferroelectrics", Physical Review, Vol. 116/1, 1959, pp. 61-68

[9] M. Takeo et al., " $\mathrm{SrBi}_{2} \mathrm{Ta}_{2} \mathrm{O}_{9}$ Thin Film Capacitor Model Including Polarization Reversal Response for Nanosecond Range Circuit Simulation of Ferroelectric Nonvolatile Memory", IEDM 1997, pp.621-624

[10] D. Rep and M. Prins, "Equivalent-Circuit Modeling of Ferroelectric Switching Devices", Journal of Appl. Phys., Vol. 85/11, 1999, pp. 7923-7930

[11] H. Wieder, "Model for Switching and Polarization Reversal in Colemanite", Journal of Appl. Phys., Vol. 31/1, 1960, pp. 180-187 